\title{
ATIVIDADE INSETICIDA DE ÓLEOS ESSENCIAIS E FIXOS SOBRE Callosobruchus maculatus (FABR., 1775) (Coleoptera: Bruchidae) EM GRÃOS DE CAUPI [Vigna unguiculata (L.) WALP.]
}

\author{
Insecticide activity of essential and fixed oils in Callosobruchus maculatus (FABR., 1775) \\ (Coleoptera: Bruchidae) in cowpea grains [Vigna unguiculata (L.) WALP.]
}

\author{
Adriana Carla Ribeiro Lopes Pereira ${ }^{1}$, José Vargas de Oliveira $^{2}$, \\ Manoel Guedes Corrêa Gondim Junior ${ }^{3}$, Cláudio Augusto Gomes da Câmara ${ }^{4}$
}

\begin{abstract}
RESUMO
O caruncho, Callosobruchus maculatus (Fabr.), é considerado a praga mais importante do caupi, Vigna unguiculata (L.) Walp., armazenado em regiões tropicais e subtropicais. Visando minimizar os efeitos indesejáveis dos inseticidas químicos sintéticos, o controle dessa praga com óleos de origem vegetal vem se constituindo numa alternativa promissora, de baixo custo e segura para os aplicadores e consumidores. Foram testados os óleos essenciais [(Cymbopogon martini (Roxb.) J.F. Watson], Piper aduncum L., Piper hispidinervum C.DC., Melaleuca sp., Lippia gracillis Shauer) e fixos (Helianthus annuus L, Sesamum indicum L, Gossypium hirsutum L., Glycine max (L.) Merr. e Caryocar brasiliense Camb.), em grãos de caupi, cv. Sempre Verde. Os óleos foram utilizados nas concentrações 10, 20, 30, 40 e $50 \mu \mathrm{L} / 20 \mathrm{~g}$, correspondendo a $0,5,1,0,1,5,2,0$ e 2,5 L/t e impregnados aos grãos no interior de recipientes de plástico, com auxílio de pipetador automático e agitados manualmente durante dois minutos. Parcelas de $20 \mathrm{~g}$ de caupi foram infestadas com oito fêmeas de $C$. maculatus, com 0 a $48 \mathrm{~h}$ de idade. Cada óleo foi testado, separadamente, em delineamento inteiramente casualisado com seis repetições. Os óleos essenciais de C. martini, $P$. aduncum e L. gracillis causaram $100 \%$ de mortalidade em todas as concentrações, $P$. hispidinervum a partir de 1,5 L/t e Melaleuca sp. nas concentrações de 2,0 e 2,5 L/t. A redução do número de ovos viáveis e de insetos emergidos foi de 100\% para todos os óleos essenciais, exceto Melaleuca sp. Por outro lado, os óleos fixos, apesar de apresentarem baixa mortalidade em todas as concentrações testadas, reduziram em praticamente $100 \%$ o número de ovos viáveis e de insetos emergidos.
\end{abstract}

Termos para indexação: Caruncho do caupi, óleos vegetais, toxicidade, efeito inseticida, Callosobruchus maculatus, Vigna unguiculata.

\section{ABSTRACT}

The weevil Callosobruchus maculatus (Fabr.) is considered the most important pest of cowpea, Vigna unguiculata (L.) Walp., stored in tropical and subtropical regions. Focusing to minimize the undesired effects of synthetic chemical insecticides, vegetal origin oils are a promissory alternative to control it because of its low cost and safety to the applicator and consumers. Essential oils [(Cymbopogon martini (Roxb.) J.F. Watson], Piper aduncum L., Piper hispidinervum C.DC., Melaleuca sp. and Lippia gracillis Shauer) and fixed oils (Helianthus annuus L., Sesamum indicum L., Gossypium hirsutum L., Glycine max (L.) Merr. and Caryocar brasiliense Camb.) were tested in cowpea grains (cv. "Sempre Verde"). These oils were used in concentrations of 10, 20, 30, 40 and 50 $\mathrm{LL} / 20 \mathrm{~g}$, corresponding to $0.5,1.0,1.5,2.0$ and $2.5 \mathrm{~L} / \mathrm{t}$ and impregnated to the grains into the plastic recipients using an automatic pipette and handly agitated for two minutes. Plots of $20 \mathrm{~g}$ from cowpea were infested with eight females of $C$. maculatus from 0 to 48 hours old. Each oil was tested separately in a randomly arrangement with six replicates. The essential oils of C. martini, P. aduncum and L. gracillis provoked $100 \%$ of mortality in all concentrations, P. hispedinervum from $1.5 \mathrm{~L} / \mathrm{t}$ and Melaleuca sp. in the concentrations of 2.0 and 2.5 L/t. The reduction of the viable eggs and emerged insects was about $100 \%$, except for Melaleuca sp. On the other hand, the fixed oils, in spite of its low mortality in all tested concentrations, reduced to almost 100\% the number of the viable eggs and emerged insects.

Index terms: Cowpea weevil, vegetal oils, toxicity, insecticide effect, Callosobruchus maculatus, Vigna unguiculata.

(Recebido em 3 de outubro de 2006 e aprovado em 18 de julho de 2007)

\section{INTRODUÇÃO}

O caruncho, Callosobruchus maculatus (Fabr., 1775) (Coleoptera: Bruchidae), é considerado a praga mais relevante do caupi [Vigna unguiculata (L). Walp.] armazenado, em regiões tropicais e subtropicais, podendo causar perdas da ordem de 60\% (TANZUBIL, 1991). Os danos causados são decorrentes da colonização do interior dos grãos pelas larvas do inseto, resultando em perda de peso, desvalorização comercial, redução do valor nutritivo,

\footnotetext{
'Bióloga, Mestre em Entomologia Agrícola - Departamento de Agronomia/DEPA - Universidade Federal Rural de Pernambuco/UFRPE - Avenida Dom Manoel de Medeiros s/n - Dois Irmãos - 52171-900 - Recife, PE - acrl@bol.com.br

²Engenheiro Agrônomo, Doutor em Entomologia - Departamento de Agronomia/DEPA - Universidade Federal Rural de Pernambuco/UFRPE - Avenida Dom Manoel de Medeiros s/n - Dois Irmãos - 52171-900 - Recife, PE - vargasoliveira@uol.com.br

${ }^{3}$ Engenheiro Agrônomo, Doutor em Entomologia - Departamento de Agronomia/DEPA - Universidade Federal Rural de Pernambuco/UFRPE - Avenida Dom Manoel de Medeiros s/n - Dois Irmãos - 52171-900 - Recife, PE - mguedes@depa.ufrpe.br

${ }^{4}$ Químico, Doutor em Química - Departamento de Química/DQ - Universidade Federal Rural de Pernambuco/UFRPE - Avenida Dom Manoel de Medeiros s/n - Dois Irmãos - 52171-900 - Recife, PE - camara@dq.ufrpe.br
} 
do grau de higiene do produto pela presença de excrementos, ovos e insetos e do poder germinativo das sementes (ALMEIDA et al., 2005; GALLO et al., 2002).

A crescente preocupação da sociedade em relação aos efeitos colaterais dos agrotóxicos, como a toxicidade para os aplicadores, poluição ambiental e a presença de resíduos em alimentos, tem incentivado os pesquisadores a desenvolverem estudos com novas táticas de controle alternativo de pragas, como o uso de inseticidas de origem vegetal (ALMEIDA et al., 2004; TAVARES \& VENDRAMIM, 2005). Plantas com atividade inseticida são ricas em compostos secundários, destacando-se os monoterpenos e seus análogos, que são compostos tipicamente lipofílicos, tendo alto potencial para interferências tóxicas em processos bioquímicos básicos, com conseqüências fisiológicas e comportamentais em insetos (PRATES \& SANTOS, 2002).

Os inseticidas de origem vegetal (pós, óleos e extratos) são considerados promissores para o manejo integrado de $C$. maculatus nas unidades de armazenamento, atuando por contato, ingestão e fumigação (ALMEIDA et al., 2005; BRITO et al., 2006; SOUSA et al., 2005). Provocam mortalidade, repelência, efeitos no crescimento, redução na oviposição e na emergência de adultos (BOEKE et al., 2004; KETOH et al., 2005; LALE \& ABDULRAHMAN, 1999; PASCUAL-VILLALOBOS \& BALLESTA-ACOSTA, 2003). Geralmente possuem baixa toxicidade para o homem e animais, baixo custo e são fáceis de serem adquiridos e utilizados pelos produtores.

Objetivou-se, neste trabalho, avaliar a atividade inseticida de óleos essenciais e fixos na mortalidade, número de ovos viáveis e emergência de $C$. maculatus em caupi armazenado.

\section{MATERIAL E MÉTODOS}

Os experimentos foram conduzidos no Laboratório de Entomologia Agrícola do Departamento de Agronomia da Universidade Federal Rural de Pernambuco (UFRPE), à $30 \pm 0,7^{\circ} \mathrm{C}, 67 \pm 2,7 \%$ UR e fotofase de 12 horas.

Criação de $\boldsymbol{C}$. maculatus: Os insetos foram criados em grãos de caupi, cv. Sempre Verde, acondicionados em recipientes de vidro de $100 \mathrm{~g}$ de capacidade, fechados com tampa plástica perfurada e revestida internamente com tecido fino. Foram confinados durante quatro dias para efetuarem a postura, em seguida retirados e os recipientes estocados até a emergência da geração $F_{1}$. Esse procedimento foi efetuado por sucessivas gerações, de modo a assegurar a quantidade de adultos necessários para a execução dos experimentos.
Eliminação da infestação e equilíbrio da umidade dos grãos. Grãos de caupi limpos e secos, utilizados para a criação e experimentos, foram acondicionados em sacos plásticos e mantidos em freezer sob temperatura de $-10^{\circ} \mathrm{C}$, durante sete dias, para eliminação de eventuais infestações de insetos provenientes do campo. Em seguida, os grãos foram transferidos para frascos de vidro de 2 L e mantidos no laboratório à temperatura ambiente durante dez dias com a finalidade de atingirem o equilíbrio higroscópico.

Obtenção dos óleos essenciais e fixos. Os óleos essenciais de palmarosa (Cymbopogon martini), de pimenta-de-macaco (Piper aduncum), de pimenta longa (Piper hispidinervum), de melaleuca (Melaleuca sp.) e de alecrim (Lippia gracillis) foram procedentes, respectivamente, da Aromalandia, Belo Horizonte, MG., do CEPLAC, Belém, PA, da Embrapa Acre, Rio Branco, AC, do Laboratório Larix, Viçosa, MG e do Laboratório de Produtos Naturais Bioativos do Departamento de Química da UFRPE, Recife, PE. Os óleos fixos de girassol (Helianthus annuus), de gergelim (Sesamum indicum), de algodão (Gossypium hirsutum), de soja (Glycine max) e de pequi (Caryocar brasiliense) foram provenientes de empresas de óleos vegetais e adquiridos no comércio da cidade do Recife - PE.

Aplicação dos óleos essenciais e fixos. Os óleos foram testados nas concentrações $10,20,30,40$ e $50 \mu \mathrm{L} /$ $20 \mathrm{~g}$, correspondentes a $0,5,1,0,1,5,2,0$ e $2,5 \mathrm{~L} / \mathrm{t}$. A impregnação dos óleos aos grãos de caupi, cv. Sempre Verde, foi efetuada no interior de recipientes de plástico, com o auxílio de pipetador automático e submetidos à agitação manual durante dois minutos. Cada parcela de $20 \mathrm{~g}$ de grãos, acondicionados em recipiente de plástico de capacidade de $120 \mathrm{ml}$, com tampa perfurada e revestida internamente com tecido fino, foi infestada com oito fêmeas de $C$. maculatus com 0 a 48 horas de emergidas, as quais permaneceram confinadas durante quatro dias.

Cada óleo foi testado, separadamente, no delineamento experimental inteiramente casualizado, constando de seis concentrações e seis repetições. A avaliação da atividade inseticida foi efetuada por meio da contagem do número de insetos mortos no quarto dia, número de ovos viáveis em todos os grãos da parcela aos 12 dias, e número de insetos emergidos aos 32 dias após a infestação. Os resultados de mortalidade foram transformados em arc seno $\sqrt{ } \%$ e os demais em $\mathrm{x}+1$, submetidos à análise de variância e as médias comparadas pelo teste de Tukey $(\mathrm{P} \leq 0,05)$, mediante a utilização do programa Sanest 3.0 (ZONTA et al., 1986). 


\section{RESULTADOS E DISCUSSÃO}

Os óleos essenciais, quando aplicados nas concentrações de 0,5 a 2,5 L/t de grãos de caupi foram mais eficientes, em relação aos óleos fixos, na mortalidade de adultos de C. maculatus (Figura 1). C. martini, P. aduncum e $L$. gracillis causaram mortalidade de $100 \% \mathrm{em}$ todas as concentrações utilizadas; $P$. hispidinervum provocou mortalidade entre $91,6 \%$ e $100 \%$ nas concentrações acima de 1,0 L/t, enquanto Melaleuca sp. causou mortalidade próximo a $100 \%$ apenas nas concentrações 2,0 e 2,5 L/t. A mortalidade provocada pelos óleos fixos de $H$. annuus., S. indicum., G. hirsutum, G. max e C. brasiliense variou entre 6,25 (0,5 L/t) a $68,75 \%(2,5 \mathrm{~L} /$ t). Não houve mortalidade natural na testemunha, com exceção do bioensaio com o óleo de Melaleuca sp., cujo porcentual de mortalidade foi de $2,0 \%$.

Os óleos essenciais e fixos foram efetivos na redução do número de ovos viáveis e de adultos de $C$. maculatus emergidos (Figura 2 e 3), apesar do óleo de Melaleuca sp. ter sido eficiente apenas nas maiores dosagens e os óleos fixos terem apresentado mortalidade mais baixa (Figura 1). Esse fato demonstra que nos estudos com óleos vegetais, a mortalidade dos insetos nem sempre é muito efetiva, de vez que esses produtos, ao contrário dos inseticidas tradicionais, atuam mais como repelentes, deterrentes de alimentação e oviposição, reguladores de crescimento, etc, caracterizando mais um efeito insetistático do que inseticida (ALMEIDA et al., 2005; DON PEDRO, 1989; OLIVEIRA \& VENDRAMIM, 1999).

Os resultados indicam que os óleos essenciais provocaram alta mortalidade por contato e, conseqüentemente, redução significativa no número de ovos viáveis e de adultos de C. maculatus emergidos; os óleos fixos, por sua vez, apresentaram menor ação de contato, mas um efeito ovicida/larvicida expressivo, reduzindo a emergência de adultos. Don Pedro (1989) postulou que a morte do ovo em bruquídeos do feijão armazenado decorre da falta de atividade respiratória suficiente, acumulação de metabólitos tóxicos e toxicidade direta devido à penetração dos óleos ou dos seus constituintes no interior do mesmo. Credland (1992) demonstrou que, entre o ovo de Callosobruchus spp. e a testa da semente, na qual o mesmo é aderido, existe um espaço interno conectado com o exterior através de uma abertura denominada "funil". A oclusão do "funil" por alguns óleos poderia explicar a razão dos seus efeitos ovicida e talvez larvicida, bem como a maior suscetibilidade dos ovos de Callosobruchus spp. a óleos vegetais, em relação a outros bruquídeos, que não possuem o "funil".
O grau de saturação dos óleos também pode ter contribuído para a sua eficiência, pois de acordo com Hall \& Harmann (1991), os lipídios altamente insaturados penetram na semente através da testa e se acumulam na superfície dos cotilédones, e os saturados não penetram, formando uma película mais espessa sobre o ovo, sendo, desse modo, mais eficientes no controle.

Segundo Seri-Kouassiet al. (2004), o óleo essencial de Melaleuca quinquenervia (Cav.) S.T. Blake foi efetivo no controle de adultos de $C$. maculatus, via fumigação, com $\mathrm{DL}_{50}$ de 3,09 $\mu \mathrm{L} / \mathrm{L}$ de ar, sendo os compostos majoritários eucaliptol (52\%) e limonene (13\%), considerados os principais responsáveis pela ação inseticida. Várias espécies de Melaleuca exerceram ação fumigante contra adultos de Sitophilus oryzae L., com valores de $\mathrm{DL}_{50}$ variando entre 28,6 a > 50 $\mu \mathrm{L} / \mathrm{L}$ de ar (LEE et al., 2004). C. martini e $P$. aduncum nas concentrações entre 0,5 a 2,5 L/t de grãos de Phaseolus vulgaris $\mathrm{L}$. provocaram $100 \%$ de mortalidade e total redução do número de ovos viáveis e de adultos de Zabrotes subfasciatus (Boh.) emergidos (S.M. de França, dados não publicados), sendo esses resultados semelhantes aos aqui obtidos. Folhas de $P$. hispidinervum contêm $94,72 \%$ de safrol, como composto majoritário, e as de P. aduncum, $73,97 \%$ de dilapiol, $3,92 \%$ de safrol e $2,84 \%$ de sarisan. Os óleos dessas plantas apresentaram $\mathrm{CL}_{50}$ de 0,51 e $2,87 \mu \mathrm{L} / \mathrm{cm}^{2}$ para adultos de Sitophilus zeamais Motsch., respectivamente (ESTRELA et al., 2006). O óleo de $L$. gracillis causou $100 \%$ de mortalidade e de redução da emergência de adultos de $S$. zeamais em milho armazenado (COITINHO, 2004), coincidindo com os resultados obtidos nesse trabalho.

O óleo fixo de G. max reduziu a oviposição e a emergência de $C$. maculatus, em grãos- de-bico, com o aumento da concentração utilizada (PACHECO et al., 1995). Os efeitos sobre ovos e/ou larvas foram, provavelmente, decorrentes da toxicidade e/ou de propriedades físicas do óleo, conforme mencionado por Don Pedro (1989). Arruda \& Batista (1998) verificaram que os óleos de G. max e $H$. annuus foram eficazes na redução da oviposição e da emergência de $C$. maculatus e o óleo de $S$. indicum inibiu a fecundidade (VARNA \& PANDEY, 1978). Os óleos de $H$. annuus e $S$. indicum na concentração de $10 \mathrm{ml} / \mathrm{Kg}$ reduziram, significativamente, a oviposição e a longevidade de adultos de $C$. maculatus em caupi (RAJAPAKSE \& EMDEN, 1997).

Os óleos essenciais e fixos podem ser de grande valia, como uma tática alternativa aos inseticidas químicos sintéticos nos programas de manejo integrado de $C$. maculatus, em unidades de armazenamento do caupi. 
Óleos essenciais

C. martini

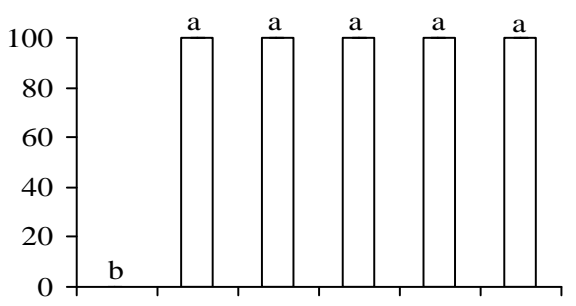

P. aducum

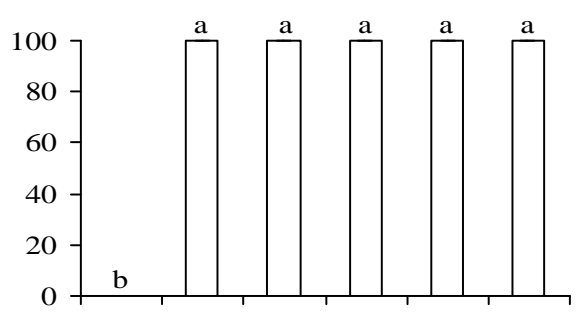

P. hispidinervum
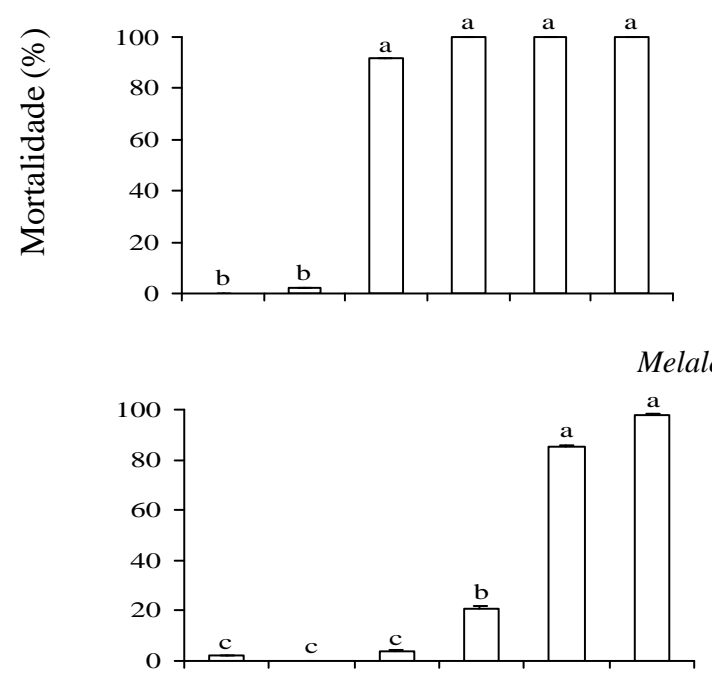

L. gracillis

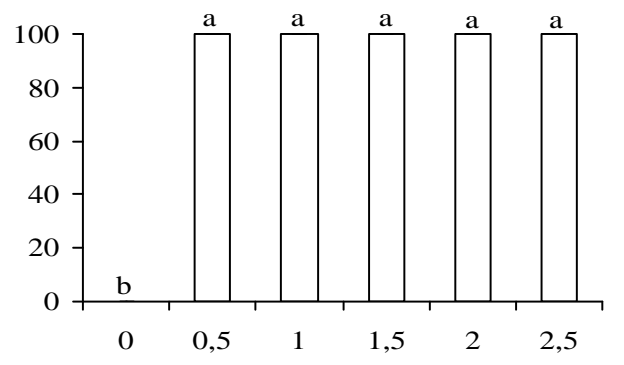

Óleos fixos

H. annuus

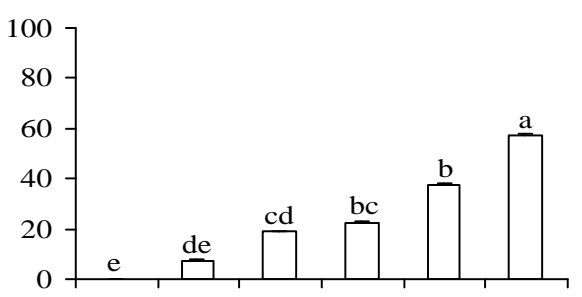

S. indicum

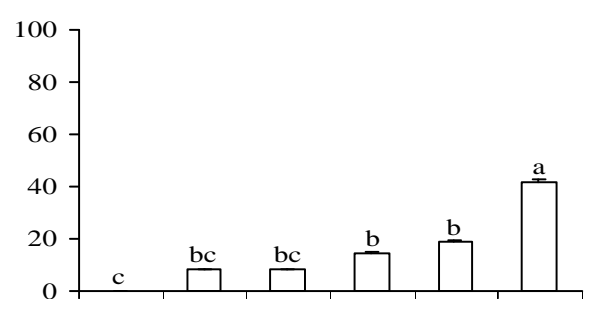

G. hirsutum
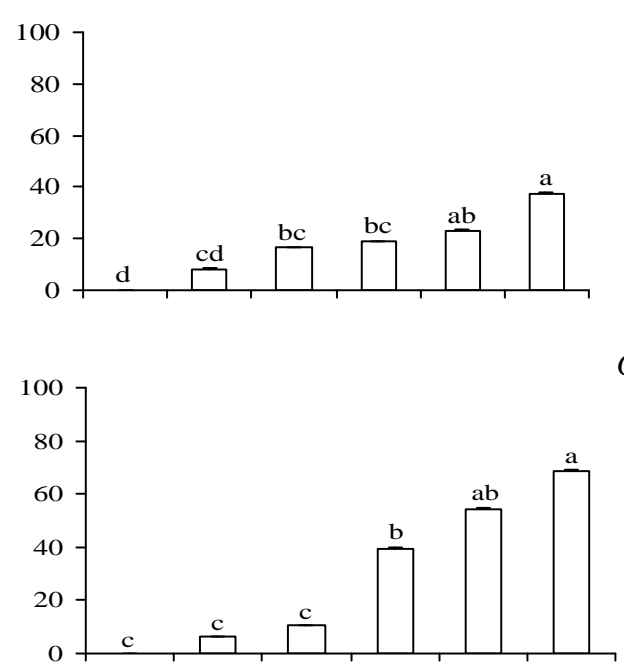

C. brasiliense

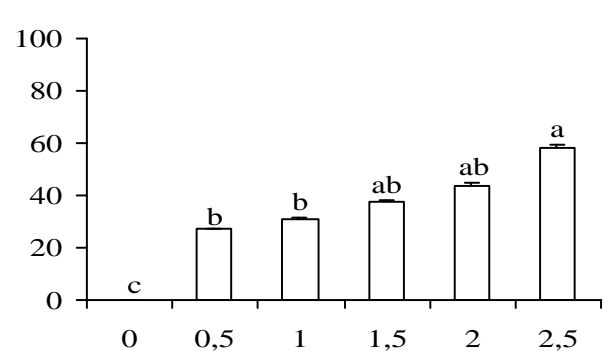

Concentrações (L/t)

Figura 1 - Porcentagens de mortalidade (média $\pm \mathrm{EP}$ ) de C. maculatus em grãos de caupi, V. unguiculata, tratados com óleos essenciais e fixos. Colunas contendo a mesma letra não diferem entre si pelo teste de Tukey $(\mathrm{P} \leq 0,05)$. 
Óleos essenciais

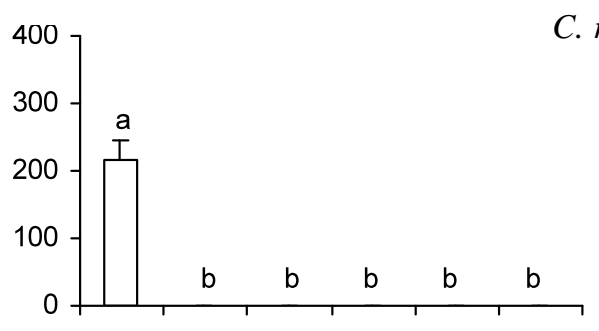

Óleos fixos
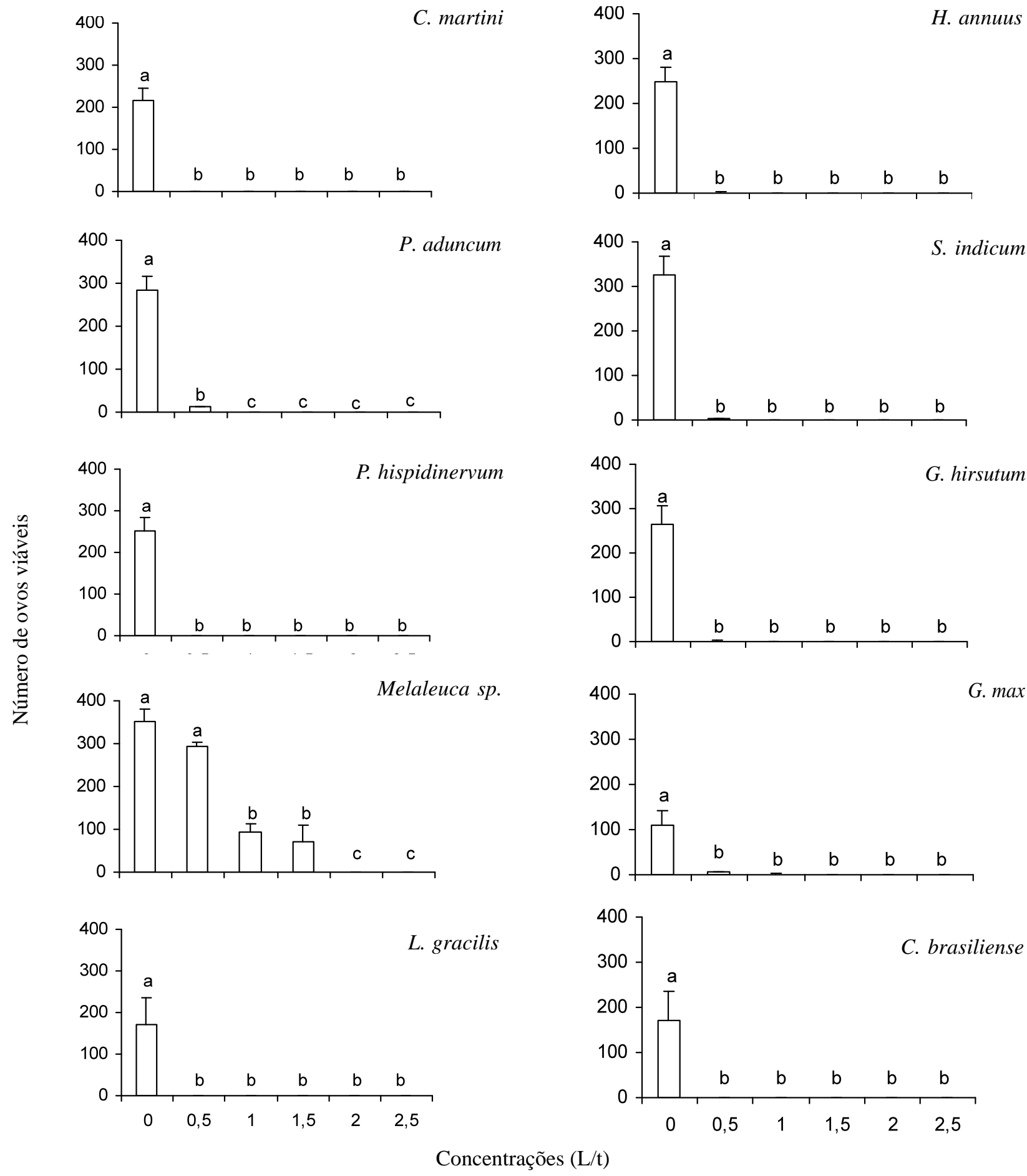

Figura 2 - Número de ovos viáveis (média \pm EP) de C. maculatus em grãos caupi, $V$. unguiculata, tratados com óleos essenciais e fixos. Colunas contendo a mesma letra não diferem entre si pelo teste de Tukey $(\mathrm{P} \leq 0,05)$. 
Óleos essenciais
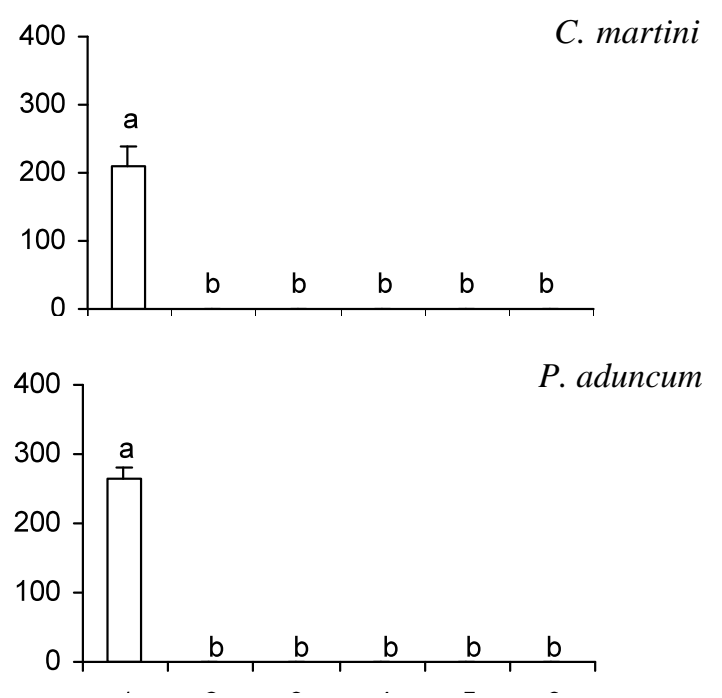

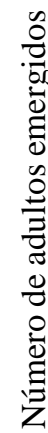
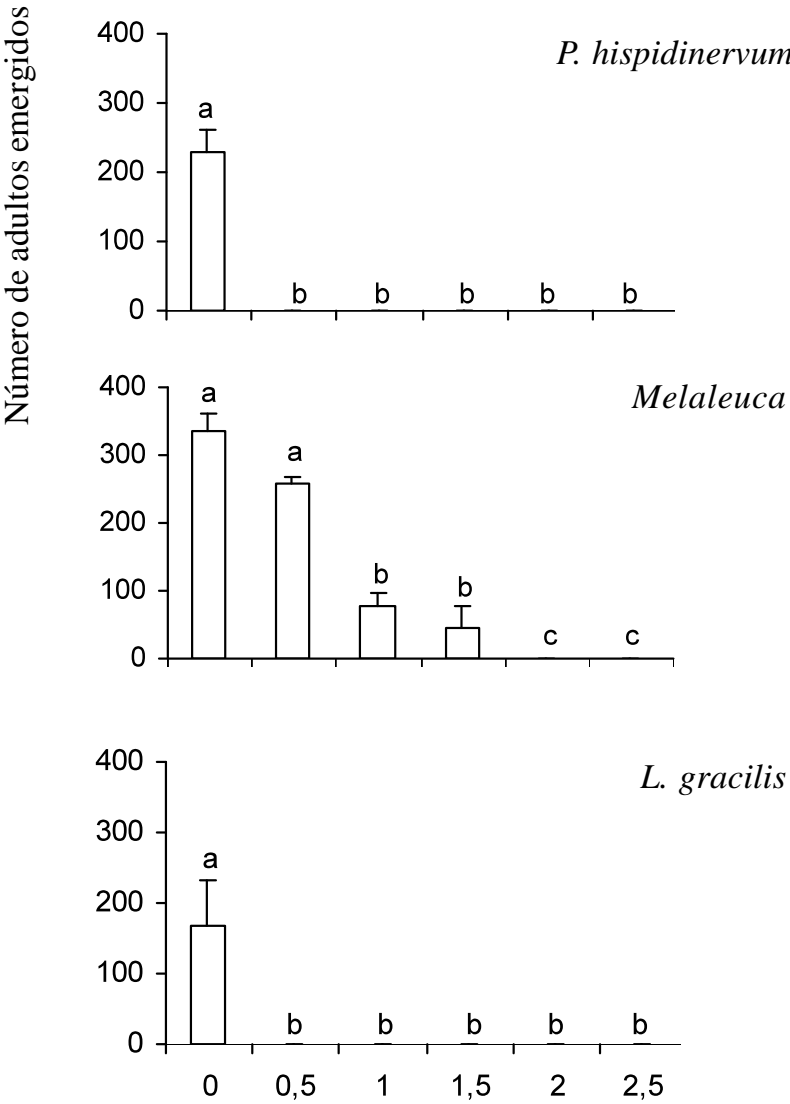

Óleos fixos
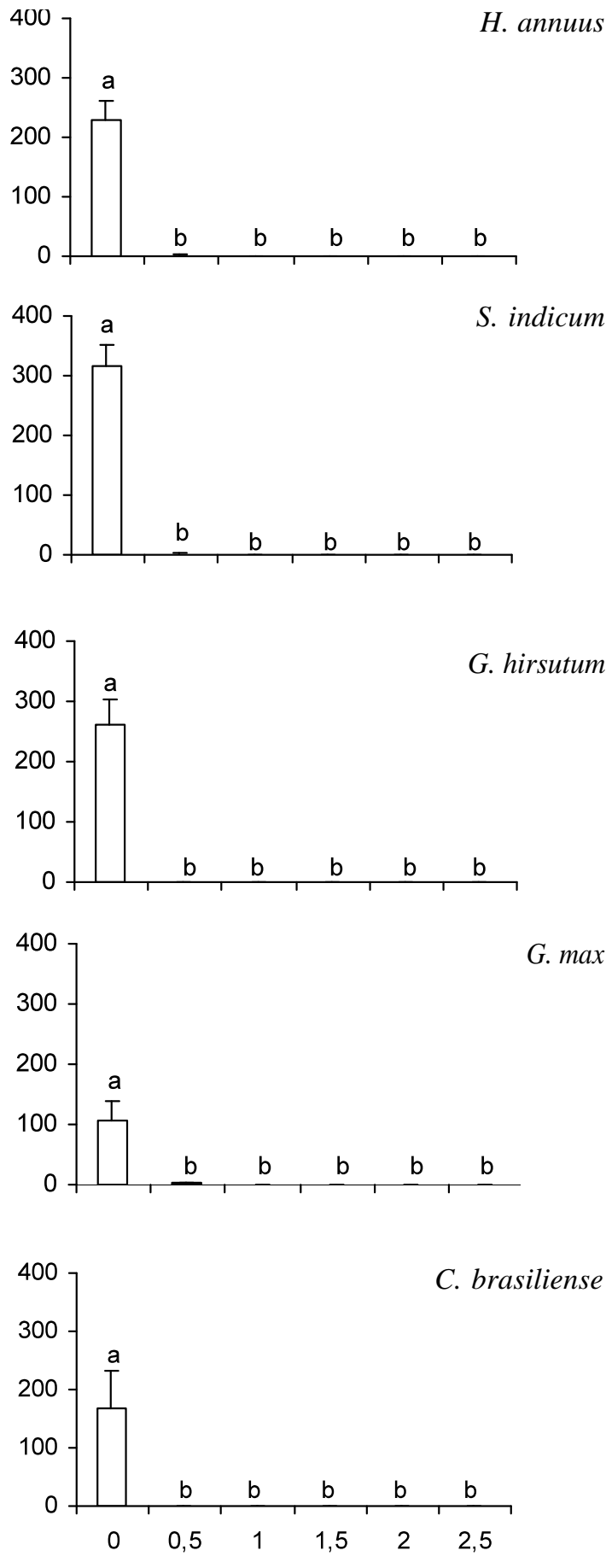

Concentrações (L/t)

Figura 3 - Número de adultos (média \pm EP) de C. maculatus emergidos em grãos de caupi, $V$. unguiculata, tratados com óleos essenciais e fixos. Colunas contendo a mesma letra não diferem entre si pelo teste de Tukey $(\mathrm{P} \leq 0,05)$. 


\section{CONCLUSÕES}

Os óleos essenciais são efetivos na mortalidade de adultos de $C$. maculatus, bem como na redução da postura e emergência. Os óleos fixos, por sua vez, provocam baixa mortalidade de adultos, mas são eficientes na redução do número de ovos viáveis e de adultos emergidos.

\section{AGRADECIMENTOS}

À CAPES pela bolsa concedida ao primeiro autor, junto ao Programa de Pós-Graduação em Entomologia Agrícola da UFRPE. Ao Prof. Jorge Braz Torres do Programa de Pós-Graduação em Entomologia Agrícola da UFRPE, pelas valiosas sugestões. À aluna do programa de PósGraduação em Entomologia Agrícola da UFRPE, Alicely Araújo Correia pela ajuda na confecção das Figuras.

\section{REFERÊNCIAS BIBLIOGRÁFICAS}

ALMEIDA, F. A. C.; ALMEIDA, S. A.; SANTOS, N. R.; GOMES, J. P.; ARAÚJO, M. E. R. Efeito de extratos alcoólicos de plantas sobre o caruncho do feijão vigna (Callosobruchus maculatus). Revista Brasileira de Engenharia Agrícola e Ambiental, Campina Grande, v. 9 , n. 4, p. 585-590, out. 2005.

ALMEIDA, S. A.; ALMEIDA, F. A. C.; SANTOS, N. R.; ARAÚJO, M. E. R.; RODRIGUES, J. P. Atividade inseticida de extratos vegetais sobre Callosobruchus maculatus (Fabr., 1775) (Coleoptera: Bruchidae). Revista Brasileira de Agrociência, Pelotas, v. 10, n. 1, p. 67-70, 2004.

ARRUDA, F. P.; BATISTA, J. L. Efeito da luz, de óleos vegetais e de cultivares de caupi na infestação do caruncho (Callosobruchus maculatus (Fabr., 1775) (Coleoptera: Bruchidae). Caatinga, Mossoró, v. 11, n. 1, p. 53-57, dez. 1998.

BOEKE, S. J.; BAUMGART, I. R.; LOON, J. J. A. van; HUIS, A. van; DICKE, M.; KOSSOU, D. K. Toxicity and repellence of African plants traditionally used for the protection of stored cowpea against Callosobruchus maculatus. Journal of Stored Products Research, Oxford, v. 40, n. 4, p. 423-438, 2004.

BRITO, J. P.; OLIVEIRA, J. E. M.; BORTOLI, S. A. Toxicidade de óleos essenciais de Eucalyptus spp. sobre Callosobruchus maculatus (Fabr., 1775) (Coleoptera: Bruchidae). Revista de Biologia e Ciências da Terra, Campina Grande, v. 6, n. 1, p. 96-103, 2006.
COITINHO, R. L. B. C. Bioatividade de inseticidas naturais sobre Sitophilus zeamais Mots. (Coleoptera: Curculionidae) em grãos de milho Zea mays $\mathbf{L}$. armazenados. 2004. 81 f. Dissertação (Mestrado em Fitossanidade) - Universidade Federal Rural de Pernambuco, Recife, 2004.

CREDLAND, P. F. The structure of bruchid eggs may explain the ovicidal effect of oils. Journal of Stored Products Research, Oxford, v. 28, n. 1, p. 1-9, 1992.

DON PEDRO, K. N. Mode of action of fixed oils against eggs of Callosobruchus maculatus (F.) (Coleoptera: Bruchidae). Pesticide Science, Oxford, v. 26, n. 2, p. 107116, 1989.

ESTRELA, J. L. V.; FAZOLIN, M.; CATANI, V.; ALÉCIO, M. R.; LIMA, M. S. Toxicidade de óleos essenciais de Piper aduncum e Piper hispidinervum em Sitophilus zeamais. Pesquisa Agropecuária Brasileira, Brasília, v. 41, n. 2, p. 217-222, 2006.

GALLO, D.; NAKANO, O.; SILVEIRA NETO, S.; CARVALHO, R. P. L.; BATISTA, G. C.; BERTI FILHO, E.; PARRA, J. R. P.; ZUCCHI, R. A.; ALVES, S. B.; VENDRAMIM, J. D.; MARCHINI, L. C.; LOPES, J. R. S.; OMOTO, C. Entomologia agrícola. Piracicaba: FEALQ, 2002. 920 p.

HALL, J. S.; HARMANN, G. E. Protection of stored legume seeds against attack by storage fungi and weevils: mechanism of action of lipoidal treatments. Crop Protection, Guildford, v. 10, n. 4, p. 375-380, 1991.

KETOH, G. K.; KOUMAGLO, H. K.; GLITHO, I. A. Inhibition of Callosobruchus maculatus (F.) (Coleoptera: Bruchidae) development with essential oil extracted from Cymbopogon schoenanthus L. Spreng. (Poaceae), and the wasp Dinarmus basalis (Rondani) (Hymenoptera: Pteromalidae). Journal of Stored Products Research, Oxford, v. 41, n. 4, p. 363-371, 2005.

LALE, N. E. S.; ABDULRAHMAN, H. T. Evaluation of neem (Azadirachta indica A. Juss.) seed oil obtained by different methods and neem powder for the management of Callosobruchus maculatus (F.) (Coleoptera: Bruchidae) in stored cowpea. Journal of Stored Products Research, Oxford, v. 35, n. 2, p. 135-143, 1999. 
LEE, B. H.; ANNIS, P. C.; TURMAALII, F.; WON-SIK, C. Fumigant toxicity of essential oils from the Myrtaceae family and 1,8-cineole against 3 major stored-grain insects. Journal of Stored Products Research, Oxford, v. 40, n. 5, p. 553-564, 2004.

OLIVEIRA, J. V.; VENDRAMIM, J. D. Repelência de óleos essenciais e pós vegetais sobre adultos de Zabrotes subfasciatus (Boh.) (Coleoptera: Bruchidae) em sementes de feijoeiro. Anais da Sociedade Entomológica do Brasil, Londrina, v. 28, n. 3, p. 549-555, abr./set. 1999.

PACHECO, I. A.; CASTRO, M. F. P. P. M.; PAULA, D. C.; LOURENÇÃO, A. L.; BOLONHEZI, S.; BARBIERI, M. K. Efficacy of soybean and castor oils in the control of Callosobruchus maculatus (F.) and Callosobruchus phaseoli (Gyllenhal) in stored chick-peas (Cicer arietinum L.). Journal of Stored Products Research, Oxford, v. 31, n. 3, p. 221-228, jul. 1995.

PASCUAL-VILLALOBOS, M. J.; BALLESTA-ACOSTA, M. C. Chemical variation in an Ocimum basilicum germplasm collection and activity of the essential oils on Callosobruchus maculatus. Biochemical Systematics and Ecology, Oxford, v. 31, n. 7, p. 673-679, July 2003.

PRATES, H. T.; SANTOS, J. P. Óleos essenciais no controle de pragas de grãos armazenados. In: LORINI, I.; MIIKE, L. H.; SENSSEL, V. M. (Eds.). Armazenagem de grãos. Campinas: Instituto Bio Geneziz, 2002. p. 443-461, 1000 p.

RAJAPAKSE, R.; EMDEN, H. F. van. Potential of four vegetable oils and ten botanical powders for reducing infestation of cowpeas by Callosobruchus maculatus, C.chinesis and C.rhodesianus. Journal of Stored Products Research, Oxford, v. 33, n. 1, p. 59-68, Jan. 1997.
SERI-KOUASSI, B. P.; KANKO, C.; ABOUA, L. R. N.; BEKON, K. A.; GLITHO, A. I.; KOUKOUA, G.; N'GUESSAN, Y. T. Action des huiles essentielles de deux plantes aromatiques de Côte-d'Ivoire sur Callosobruchus maculatus F. du niébé. Comptes Rendus Chimie, Paris, v. 7, n. 10/11, p. 1043-1046, 2004

SOUSA, A. H.; MARACAJÁ, P. B.; SILVA, R. M. A.; MOURA, A. M. N.; ANDRADE, W. G. Bioactivity of vegetal powders against Callosobruchus maculatus (Coleoptera: Bruchidae) in caupi bean and seed physiological analysis. Revista de Biologia e Ciencias da Terra, Campina Grande, v. 5, n. 2, 2005. Disponível em: <http://www.uepb.edu.br/eduep/rbct/sumarios/ sumario_v5_n2.htm>. Acesso em: 25 jul. 2006.

TANZUBIL, P. B. Control of some insect pests of cowpea (Vigna unguiculata) with neem (Azadirachta indica) in Northern Ghana. Tropical Pest Management, Basingstoke, v. 37, n. 3, p. 216-217, July/Sept. 1991.

TAVARES, M. A. G. C.; VENDRAMIM, J. D. Bioatividade da Erva-de-Santa-Maria, Chenopodium ambrosioides L., sobre Sitophilus zeamais Mots. (Coleoptera: Curculionidae). Neotropical Entomology, Londrina, v. 34, n. 2, p. 319-323, Mar./Apr. 2005.

VARMA, B. K.; PANDEY, G. P. Treatment of stored greengram seeds with edible oils for protection from Callosobruchus maculatus (Fabr.). Indian Journal of Agricultural Science, New Delhi, v. 48, n. 2, p. 72-75, 1978.

ZONTA, E. P.; SILVEIRA, P.; MACHADO, A. A. Sistema de análise estatística (SANEST 3.0). Pelotas: UFPel, 1986. 399 p. 